Pacific Journal of Mathematics

CLIFFORD'S THEOREM FOR ALGEBRAIC GROUPS AND LIE
ALGEBRAS

JOHN BALAS 


\title{
CLIFFORD'S THEOREM FOR ALGEBRAIC GROUPS AND LIE ALGEBRAS
}

\author{
JOHN W. BALLARD
}

\begin{abstract}
The standard results for comparing the irreducible representations of a group to those of a normal subgroup were obtained by $\mathbf{A}$. $\mathrm{H}$. Clifford. The object of this paper is to discuss a variation of these results in which the group is assumed to be an affine algebraic group and the role of the normal subgroup is played by the Lie algebra.
\end{abstract}

1. Introduction. Let $G$ be an affine algebraic group over an algebraically closed field of positive characteristic. Let $V$ be an irreducible rational representation space for $G$. We show that when viewed as a representation space for the Lie algebra $g, V$ decomposes as the direct sum of isomorphic irreducible representations. This is the analog of the first two results in [4]. Let $W$ be an irreducible $g$-subspace of $V$. We show that $V$ factors as the tensor product of two rational projective representations of $G$, one of which is induced by $W$ while the other is a representation of the quotient group $G / \mathrm{g}$. Since the field has positive characteristic it follows that $V=W \otimes U$, where $U$ is the Frobenius power of a projective representation of $G$. This is the analog of Clifford's Theorem 3. We assume that the Lie algebra has no non-trivial one dimensional restricted representations and show that this factorization may be continued to express $V$ as the tensor product of irreducible representations of $g$ and their Frobenius powers. The Curtis-Steinberg decomposition [3] for the irreducible representations of a simply connected semisimple group then follows as a corollary.

In the course of the discussion we construct a Schur representation group $G^{s}$ of $G$ relative to its Lie algebra and a relative Schur multiplier. Essentially $G^{s}$ is the smallest covering group of $G$ which linearizes the irreducible representations of $\mathrm{g}$. Our identification of $G^{s}$ as the simply connected covering group of $G$ answers a question originally posed by Curtis [6, p. 325] in the context of Chevalley groups. We identify the relative Schur multiplier with the Picard group of the algebraic variety $G$. This amounts to showing that the Picard group is generated by the irreducible representations of the Lie algebra which do not arise as the differential of a representation for the group. 
2. Preliminaries. Let $K$ be an algebraically closed field of characteristic $p$ and let $G$ be a connected affine algebraic group defined over the prime subfield of $K$. We identify $G$ with its group of $K$-rational points and denote by $g$ or $L(G)$ the Lie algebra.

Let $A=K[G]$ be the coordinate ring of $G$ and let $A^{*}$ denote the linear dual. If $V$ is a rational $G$-module with comodule map $\Delta_{V}: V \rightarrow V \otimes$ $A$, then $V$ is a module for the algebra $A^{*}$ with the action of $X \in A^{*}$ given by $(1 \otimes X) \Delta_{V}$.

Let $M$ be the kernel of the augmentation map of $A$ and let $M_{n}$ denote the ideal of $A$ generated by $m^{q}\left(q=p^{n}, m \in M\right)$. The $n$th infinitesimal hyperalgebra $u_{n}$ of $G$ is the finite dimensional Hopf algebra $\left(A / M_{n}\right)^{*}$. So $u_{1}$ is the restricted universal enveloping algebra of $g$ while $u_{n}$ is the algebra of invariant differential operators on $A$ of order $\leq p^{n}$. We let $\Delta$ denote, generically; the comultiplication map of $u_{n}$. The tensor product of two $u_{n}$-modules is also a $u_{n}$-module, with the action of $X \in u_{n}$ given by $\Delta(X)$. The hyperalgebra of $G$ is the union of the $u_{n}$. If $\tau: G \rightarrow H$ is a morphism of algebraic groups, let $h y(\tau)$ denote the corresponding map.of hyperalgebras.

If $a_{1}, \ldots, a_{m}$ is a $K$-basis for $M / M^{2}$, then the monomials $a_{1}^{\alpha_{1}} \cdots a_{m}^{\alpha_{m}}$ $\left(0 \leq \alpha_{i}<p^{n}\right)$ form a $K$-basis for $A / M_{n}[13]$. Let $X_{i}^{(\alpha)} \in u_{n}$ be dual to $a_{i}^{\alpha}$. Then

$$
\Delta\left(X_{i}^{(\alpha)}\right)=\sum_{j=0}^{\alpha} X_{i}^{(\alpha-j)} \otimes X_{i}^{(j)}
$$

and so $\left\{X_{i}^{(\alpha)} \mid 0 \leq \alpha<p^{n}\right\}$ is a sequence of divided powers over $X_{i}^{(1)}=X_{i}$. The monomials $X_{1}^{\left(\alpha_{1}\right)} \cdots X_{m}^{\left(\alpha_{m}\right)}\left(0 \leq \alpha_{i}<p^{n}\right)$ form a $K$-basis for $u_{n}$.

Let $V$ be a rational $G$-module. Since we may view $u_{n}$ as the subalgebra of $A^{*}$ vanishing on $M_{n}, V$ is also a module for $u_{n}$, which we denote by $V \mid u_{n}$. If $\pi$ denotes the action of $G$ on $V$, we denote the action of $u_{n}$ on $V$ by $d \pi$. The relation between the adjoint action Ad of $G$ on $u_{n}$ and the action on $V$ is given by

$$
d \pi(X) \pi(g) v=\pi(g) d \pi\left(X^{g}\right) v \quad\left(X \in u_{n}, g \in G\right),
$$

where $X^{g}=\left(\operatorname{Ad~} g^{-1}\right) X$.

The following argument, which is adapted from [1, p. 132], shows that every irreducible $u_{n}$-module is a submodule of a rational irreducible $G$-module. Every irreducible $u_{n}$-module $W$ is realized as a submodule of $u_{n}^{*}=A / M_{n}$. Specifically, $W$ is isomorphic to a submodule of the space spanned by the coordinate functions for $W$. Since the map $A \rightarrow A / M_{n}$ is $u_{n}$-equivariant, we may choose $u_{n}$-submodules $U, V \subseteq A$ with $U / V \cong W$. 
View $G$ as acting by left translations on $A$ and choose a finite dimensional $G$-subspace $N$ with $U \subseteq N$. Since the action of $u_{n}$ on $A$ is the differential of left translation, $U$ and $V$ are $u_{n}$-submodules of $N \mid u_{n}$. If $m$ is the dimension of $V$, then $E^{m+1}(N)$, the $m+1$ exterior power of $N$, contains the $u_{n}$-submodule $U \wedge E^{m}(V)$. On the other hand, $U \wedge E^{m}(V)$ is $u_{n}$-isomorphic to $W \otimes E^{m}(V)$. Since $V \subset M_{n}, E^{m}(V)$ is trivial as a $u_{n}$-module. So $W$ is isomorphic to a $u_{n}$-submodule of the rational $G$-module $E^{m+1}(N)$.

We note that $u_{n}=\left(A / M_{n}\right)^{*}$ is the dual algebra for the infinitesimal group scheme $G^{n}$. Here $G^{n}$ denotes the scheme theoretic kernel for the $n$th power of the Frobenius morphism $G \rightarrow G$. The classical results for comparing the irreducible representations of a group to those of a normal subgroup were given by Clifford in [4]. The intent of this paper is to discuss the analogous results for $G$ and its normal subgroups $G^{n}$. Since $G^{n}$ has but one point over $K$, we prove the results from the equivalent viewpoint of $u_{n}$.

We begin by considering the analog of Clifford's first theorem with the role of the normal subgroup played by $u_{n}$.

(2.2) THEOREM (Clifford). Let $V$ be an irreducible rational G-module. Then

$$
V \mid u_{n}=W_{1} \oplus \cdots \oplus W_{m}
$$

where the $W_{i}$ are mutually isomorphic irreducible $u_{n}$-modules.

Proof. Let $W$ be an irreducible $u_{n}$-submodule of $V$. Since $X(g W)=$ $g\left(X^{g} W\right)\left(X \in u_{n}, g \in G\right), g W$ is also a $u_{n}$-submodule. Hence

$$
V=\sum_{g \in G} g W
$$

is a completely reducible $u_{n}$-module. Let $W_{1}, \ldots, W_{s}$ be representatives for the isomorphism classes of irreducible $u_{n}$-submodules of $V$. Let $V_{l}$ denote the sum of the $u_{n}$-submodules of $V$ which are isomorphic to $W_{i}$. Then $V=\bigoplus_{i=1}^{s} V_{i}$ and $G$ permutes the $V_{i}$, transitively since $V$ is irreducible. The stabilizer of $V_{1}$ is a closed subgroup of $G$ of index $s$ which, by connectivity, equals $G$. So $s=1$ and the result follows.

In the context of Theorem 2.2, Clifford shows that $V$ factors as the tensor product of two irreducible projective representations. By way of preparation for considering the analogous result, we conclude this section with a few elementary remarks on projective representations. 
A projective representation of $G$ on a vector space $V$ is a mapping $\pi$ : $G \rightarrow G L(V)$ such that for all $x, y \in G$

$$
\pi(x) \pi(y)=\alpha(x, y) \pi(x y) \text { and } \pi(1)=1
$$

where $\alpha: G \times G \rightarrow K^{\times}$. We refer to a projective representation with trivial cocycle as a linear representation. A projective representation is irreducible if it has no non-trivial $G$-stable subspaces. Two projective representations $\pi_{i}: G \rightarrow G L\left(V_{i}\right)(i=1,2)$ are said to be projectively equivalent if there is a linear isomorphism $\theta: V_{1} \rightarrow V_{2}$ such that for all $g \in G$

$$
\theta \pi_{1}(g)=c(g) \pi_{2}(g) \theta
$$

where $c(g)$ is a non-zero element of $K$.

We define a rational projective representation of $G$ as a projective representation $\pi: G \rightarrow G L(V)$ for which the mapping $\pi^{\prime}: G \rightarrow P G L(V)=$ $G L(V) / K^{\times}$is a morphism of algebraic varieties. The tensor product of rational projective representations is again one and the following lemma yields a form of the converse suitable for our purposes.

(2.3) LemMA. Let $\pi_{i}: G \rightarrow G L\left(V_{i}\right)(i=1,2)$ be two projective representations whose tensor product $\pi_{1} \otimes \pi_{2}: G \rightarrow G L\left(V_{1} \otimes V_{2}\right)$ is a rational projective representation. Then $\pi_{1}$ and $\pi_{2}$ are rational projective representations of G.

Proof. It suffices to show that each $\pi_{i}^{\prime}: G \rightarrow P G L\left(V_{i}\right)$ is a morphism of varieties. Let $\mathbf{P}(V)$ denote the projective space corresponding to the vector space $V$ and view $P G L(V)$ as the automorphism group of $\mathbf{P}(V)$. The Segre embedding $\mathbf{P}\left(V_{1}\right) \times \mathbf{P}\left(V_{2}\right) \rightarrow \mathbf{P}\left(V_{1} \otimes V_{2}\right)$ yields a closed immersion

$$
\rho: P G L\left(V_{1}\right) \times P G L\left(V_{2}\right) \rightarrow P G L\left(V_{1} \otimes V_{2}\right)
$$

with $\rho(a, b)=a \otimes b$. Since the comorphism for $\rho$ is surjective and $\left(\pi_{1} \otimes \pi_{2}\right)^{\prime}=\rho\left(\pi_{1}^{\prime} \times \pi_{2}^{\prime}\right)$, it follows that $\pi_{1}^{\prime} \times \pi_{2}^{\prime}$ is a rational map of $G$ into $P G L\left(V_{1}\right) \times P G L\left(V_{2}\right)$ and the result follows.

3. Clifford's Theorem. Let $m$ denote either a restricted Lie ideal of $\mathrm{g}$ which is stable under the adjoint action of $G$ or one of the infinitesimal hyperalgebras $u_{n}$. Define $P(\mathfrak{m})$, the primitive elements of $\mathfrak{m}$, as the collection of all $X \in \mathfrak{m}$ with $\Delta(X)=X \otimes 1+1 \otimes X$. With our choice of $\mathfrak{m}, P(\mathfrak{m})=\mathfrak{m}$ if $\mathfrak{m}$ is contained in $\mathfrak{g}$ and equals $\mathfrak{g}$ otherwise. Let $G / \mathfrak{m}$ denote the affine algebraic group whose coordinate ring is $A^{\mathrm{m}}$, the subalgebra of $A$ annihilated by $\mathrm{m}$ (see [2, p. 372]). The quotient morphism 
$G \rightarrow G / \mathfrak{m}$ is said to be purely inseparable since the quotient field of $A$ is a purely inseparable extension of the quotient field for $A^{\mathrm{m}}$. The following result is the analog of Clifford's Theorem 3 from [4] while our proof follows that of [7,p. 351]. We note that by Theorem 2.2 every irreducible rational $G$-module satisfies the hypothesis of the theorem.

(3.1) THEOREM (Clifford). Let $V$ be a rational G-module such that $V \mid \mathrm{m}$ is the direct sum of isomorphic irreducible $\mathfrak{m}$-modules. Let $W$ be an irreducible $\mathrm{m}$-submodule of $V$. Then there are rational projective representations

$$
\pi_{1}: G \rightarrow G L(W) \text { and } \pi_{2}: G \rightarrow G L(U)
$$

such that $\pi(g)=\pi_{1}(g) \otimes \pi_{2}(g)$ defines a rational linear representation of $G$ on $W \otimes U$ with $V \cong W \otimes U$. Moreover, $W \mid \mathrm{m}$ is projectively equivalent to $W, \pi_{1}$ is an irreducible rational projective representation of $G$ and $\pi_{2}$ is a rational projective representation of $G / \mathrm{m}$.

Proof. By assumption,

$$
V \mid \mathfrak{m}=W_{1} \oplus \cdots \oplus W_{s}
$$

where each $W_{l}$ is m-isomorphic to $W$. If $\tau$ is the representation of $m$ on $W$ and $\pi$ the representation of $G$ on $V$, then $\tau=d \pi \mid W$.

For $g \in G$, let $W^{g}$ denote the module $W$ with the action of $m$ given by

$$
X w=\tau\left(X^{g}\right) w \quad(X \in \mathfrak{m}, w \in W)
$$

Then $W^{g}$ is m-isomorphic to $\pi(g) W$ and hence isomorphic to $W$. Since $W^{g}$ has the same underlying $K$-vector space as $W$, we may choose an intertwining operator $\pi_{1}(g) \in G L(W)$ with

$$
\tau\left(X^{g}\right)=\pi_{1}(g)^{-1} \tau(X) \pi_{1}(g),
$$

for all $X \in \mathrm{m}$. For $x, y \in G$, the definition of $\pi_{1}$ shows that $\pi_{1}(x) \pi_{1}(y) \pi_{1}(x y)^{-1}$ is an m-module isomorphism of $W$. Now $W$ is irreducible and $K$ is algebraically closed, so $\pi_{1}(x) \pi_{1}(y)=\alpha(x, y) \pi_{1}(x y)$ for some $\alpha(x, y) \in K^{\times}$. Hence $\pi_{1}: G \rightarrow G L(W)$ is a projective representation of $G$.

Let $U$ be an $s$-dimensional $K$-vector space and let $f_{1}, \ldots, f_{s}$ be a basis for $U^{*}$. Choose m-module isomorphisms $\varphi_{l}: W \rightarrow W_{l}$. Then the map $\varphi$ : $W \otimes U \rightarrow V$ given by

$$
\varphi(w \otimes u)=\sum_{i=1}^{s} f_{i}(u) \varphi_{i}(w)
$$


is an isomorphism of vector spaces. We give $W \otimes U$ the structure of a rational $G$-module by requiring that $\varphi$ be a $G$-isomorphism and identify $V$ with $W \otimes U$. With this identification, $d \pi(X)=\tau(X) \otimes 1$ for all $X \in \mathfrak{m}$. Since $\pi(g)\left(\pi_{1}(g)^{-1} \otimes 1\right)$ commutes with $\tau(X) \otimes 1(X \in \mathrm{m})$, Burnside's theorem shows that it commutes with $\beta \otimes 1$, for all $\beta \in \operatorname{End}(W)$. So $\pi(g)=\pi_{1}(g) \otimes \pi_{2}(g)$ for some $\pi_{2}(g) \in G L(U)$. If $x, y \in G$, then

$$
\pi_{1}(x y) \otimes \pi_{2}(x y)=\pi(x y)=\pi(x) \pi(y)=\alpha(x, y) \pi_{1}(x y) \otimes \pi_{2}(x) \pi_{2}(y) .
$$

Hence $\pi_{2}(x) \pi_{2}(y)=\alpha(x, y)^{-1} \pi_{2}(x y)$ and so $\pi_{2}: G \rightarrow G L(U)$ is a projective representation of $G$.

Since $\pi_{1} \otimes \pi_{2}=\pi$ is a rational linear representation of $G$, Lemma 2.3 shows that $\pi_{1}$ and $\pi_{2}$ are rational projective representations of $G$.

If $X \in P(\mathfrak{m})$, then

$$
\tau^{\prime}(X) \otimes 1=d \pi^{\prime}(X)=d \pi_{1}^{\prime}(X) \otimes 1+1 \otimes d \pi_{2}^{\prime}(X),
$$

so $d \pi_{1}^{\prime} \mid P(\mathfrak{m})=\tau^{\prime}$ while $d \pi_{2}^{\prime} \mid P(\mathfrak{m})=0$. If $\mathfrak{m}=u_{n}$ and $X=X_{i}$, then

$$
\Delta\left(X^{(\alpha)}\right)=\sum_{j=0}^{\alpha} X^{(\alpha-j)} \otimes X^{(j)}
$$

for $0 \leq \alpha<p^{n}$. By induction on $\alpha$,

$$
\tau^{\prime}\left(X^{(\alpha)}\right) \otimes 1=d \pi_{1}^{\prime}\left(X^{(\alpha)}\right) \otimes 1+1 \otimes d \pi_{2}^{\prime}\left(X^{(\alpha)}\right),
$$

hence $d \pi_{1}^{\prime} \mid \mathfrak{m}=\tau^{\prime}$ and $d \pi_{2}^{\prime} \mid \mathfrak{m}=0$. Now the former shows that $W \mid \mathfrak{m}$ is projectively equivalent to the original action $\tau$ of $\mathfrak{m}$ on $W$ while the latter insures that $\pi_{2}^{\prime}$ factors through $G / \mathfrak{m}[2$, p. 376] to yield a rational projective representation of $G / \mathrm{m}$ on $U$, which completes the proof of the theorem.

As the proof of the preceding result shows, $U \cong \operatorname{Hom}_{\mathrm{m}}(W, V)$. We note that if $W$ and $U$ are linear $G$-modules, then this is a $G$-isomorphism.

For $\lambda \in \operatorname{Hom}(\mathfrak{m}, K)$, let $S(\lambda)$ denote the one dimensional $\mathfrak{m}$-module affording $\lambda$.

(3.3) CoROllaRy. In the notation of Theorem 3.1, $\pi_{1}$ and $\pi_{2}$ may be chosen to be rational linear representations of $G$ if and only if there is a rational G-module $W_{0}$ and a linear character $\lambda \in \operatorname{Hom}(m, K)$ such that $W_{0} \mid \mathfrak{m} \cong S(\lambda) \otimes W$. 
Proof. First assume that $\pi_{1}: G \rightarrow G L\left(W_{0}\right)$ and $\pi_{2}: G \rightarrow G L\left(U_{0}\right)$ are rational linear representations of $G$ such that $V \cong W_{0} \otimes U_{0}$ with $d \pi_{1}^{\prime} \mid \mathrm{m}=$ $\tau^{\prime}$ and $d \pi_{2}^{\prime} \mid \mathrm{m}=0$. Then $d \pi_{2}=\lambda$ for some $\lambda \in \operatorname{Hom}(\mathfrak{m}, K)$. Since $\tau \otimes 1$ $=d \pi, \tau(X)=\lambda(X)+d \pi_{1}(X)$, for all $X \in P(\mathfrak{m})$. If $0 \leq \alpha<p^{n}$ and $X=X_{i}$, then

$$
\begin{aligned}
\tau\left(X^{(\alpha)}\right) \otimes 1 & =d \pi\left(X^{(\alpha)}\right)=\sum_{j=0}^{\alpha} d \pi_{1}\left(X^{(\alpha-j)}\right) \otimes d \pi_{2}\left(X^{(j)}\right) \\
& =\sum_{j=0}^{\alpha} \lambda\left(X^{(j)}\right) d \pi_{1}\left(X^{(\alpha-j)}\right) \otimes 1 .
\end{aligned}
$$

So $S(\lambda) \otimes W_{0} \mid \mathfrak{m} \cong W$ and hence, denoting by $S\left(\lambda^{*}\right)$ the dual of $S(\lambda)$, $W_{0} \mid \mathfrak{m} \cong S\left(\lambda^{*}\right) \otimes W$.

Now assume that $\rho: G \rightarrow G L\left(W_{0}\right)$ is a rational linear representation of $G$ such that $W_{0} \mid m \cong S(\lambda) \otimes W$. Then

$$
\begin{aligned}
\lambda\left(X^{g}\right)+\tau\left(X^{g}\right) & =d \rho\left(X^{g}\right)=\rho(g)^{-1} d \rho(X) \rho(g) \\
& =\lambda(X)+\rho(g)^{-1} \tau(X) \rho(g),
\end{aligned}
$$

for all $X \in P(\mathfrak{m})$ and $g \in G$. Since $\lambda\left(X^{g}\right)=\lambda(X)$, we have $\tau\left(X^{g}\right)=$ $\rho(g)^{-1} \tau(X) \rho(g)$ for $X \in P(\mathrm{~m})$. It follows that $\tau\left(X^{g}\right)=\rho(g)^{-1} \tau(X) \rho(g)$ for all $X \in \mathbb{m}$ and so we may choose $\pi_{1}(g)=\rho(g)$ in equation (3.2). The linearity of $\pi_{2}$ then follows from that of $\pi$ and $\pi_{1}$.

REMARK. Let $W$ be a one dimensional $g$-module and suppose that $W$ is a submodule of an irreducible rational $G$-module $V$ with $W \neq V$. Then Corollary 3.3 shows that $V \cong W_{0} \otimes U$, where $W_{0}$ is the trivial one dimensional representation and $U$ is an irreducible rational projective representation of $G / \mathfrak{g} \cong G$. So $V \cong U$ is the Frobenius power of a rational projective representation of $G$.

(3.4) Corollary. Let $V$ be a rational G-module and let $W$ be an irreducible m-submodule. Let $O(W)$ denote the $G$-submodule generated by $W$. Then there are rational projective representations

$$
\pi_{1}: G \rightarrow G L(W) \text { and } \pi_{2}: G \rightarrow G L(U)
$$

such that $O(W) \cong W \otimes U$, an isomorphism of linear G-modules.

Proof. Since $O(W)$ is the sum of the $G$-translates of $W, O(W) \mid \mathrm{m}$ is completely reducible. Write, as in the proof of Theorem 2.2,

$$
O(W) \mid \mathfrak{m}=V_{1} \oplus \cdots \oplus V_{s}
$$


where the $V_{i}$ are the homogeneous components. The connected group $G$ permutes the $V_{i}$ transitively and so $s=1$. The result now follows from Theorem 3.1.

Denote by $g_{m}$ the Lie algebra for the multiplicative group of $K$. If $U$ is a rational $G$-module, let $U^{(n)}$ denote the $n$th Frobenius power of $U$. Suppose that $\operatorname{Hom}\left(G, G L_{1}\right)=1$ and that every irreducible rational projective representation of $G$ is equivalent to a rational linear representation. The remark following Corollary 3.3 shows that $\operatorname{Hom}\left(g, g_{m}\right)=0$. Let $V$ be an irreducible rational $G$-module. Then Theorem 3.1, with $\mathfrak{m}=\mathfrak{g}$, shows that $V=W_{0} \otimes U$, where $W_{0}$ and $U$ are uniquely determined irreducible rational $G$-modules with $W_{0} \mid \mathrm{g}$ irreducible. Since $U \mid \mathrm{g}$ is trivial, $U \cong U_{1}^{(1)}$ for some rational $G$-module $U_{1}$. Applying Theorem 3.1 to $U_{1}$ and continuing shows that

$$
V \cong W_{0} \otimes W_{1}^{(1)} \otimes \cdots \otimes W_{n}^{(n)}
$$

where the $W_{i}$ are irreducible rational $G$-modules which remain irreducible when viewed as modules for $\mathrm{g}$. So Clifford's theorem, in this context, yields the Curtis-Steinberg decomposition [3] for the irreducible modules of a simply connected semisimple group. We refer the reader to [5] for an alternate approach to this decomposition.

4. Schur representation groups. We now consider the problem of obtaining a factorization similar to that of (3.5) for the irreducible representations of a group $G$. We construct a Schur representation group $G^{s}$ of $G$ relative to its Lie algebra for which (3.5) yields a factorization of the irreducible representations of $G^{s}$ and hence a projective factorization of the irreducible representations of $G$. Since our decomposition is to be in terms of the irreducible representations of the Lie algebra, it is necessary to assume that $\operatorname{Hom}\left(\mathfrak{g}, \mathfrak{g}_{m}\right)=0$. Lacking a convenient reference, we begin the discussion by giving an elementary procedure for obtaining $P G L_{n}$ as a quotient of $S L_{n}$.

Let $n$ be a positive integer and write $n=p^{s} m$, where $(p, m)=1$. Let $T=G L_{1}$ be a one dimensional torus with coordinate ring $B=K\left[x, x^{-1}\right]$. Then $B^{\prime}=K\left[x^{n}, x^{-n}\right]$ is the coordinate ring of an affine group $T^{\prime}$ which may be obtained as a quotient of $T$ as follows.

The affine group $T / L(T)$ has coordinate ring $B^{L(T)}=K\left[x^{P}, x^{-P}\right]$. So by defining $T_{i+1}=T_{i} / L\left(T_{i}\right)$ and setting $T_{0}=T$, we obtain a sequence of purely inseparable quotient morphisms $\mu_{i}: T_{l} \rightarrow T_{i+1}$. If $X$ is a generator for $L(T)$, then the image of $X^{(q)}\left(q=p^{i}\right)$ under the corresponding 
homomorphism of hyperalgebras is a generator for $L\left(T_{t}\right)$. Now let $M$ be the finite subgroup of $m$ th roots of 1 in $T_{s}$. Then

$$
K\left[T_{s}\right]^{M}=K\left[x^{n}, x^{-n}\right]
$$

and so $T_{s} / M=T^{\prime}$. Let $\tau_{s}: T_{s} \rightarrow T^{\prime}$ be the quotient morphism. Since $\tau_{s}$ is separable, $u_{1}\left(T_{s}\right) \cong u_{1}\left(T^{\prime}\right)$ and hence $u_{1}\left(T^{\prime}\right) \cong u_{s}(T) / u_{s-1}(T)$. We show that a similar sequence of $s$ purely inseparable morphisms followed by a separable morphism yields $P G L_{n}$ as a quotient of $S L_{n}$.

Let $V$ be an $n$-dimensional vector space over $K$ and set $H=S L(V)$, $H^{\prime}=P G L(V)$. Let $\tau: H \rightarrow H^{\prime}$ be the restriction of the quotient morphism $G L(V) \rightarrow H^{\prime}$. Since $d \tau: \mathfrak{h} \rightarrow \mathfrak{h}^{\prime}$ is the natural map and $n=p^{s} m$, we note that $\operatorname{Im} d \tau$ has codimension 1 in $\mathfrak{h}^{\prime}$ if $s \neq 0$ and codimension 0 if $s=0$.

We first discuss the case where $s \neq 0$. Let $W$ denote the $G L(V)$-module obtained by tensoring the $m$ th exterior power of $V^{(s)}$ with the one dimensional module affording $\operatorname{det}^{-1}$. The representation factors through $H^{\prime}$ to yield a rational representation $H^{\prime} \rightarrow S L(W)$ for which the diagram

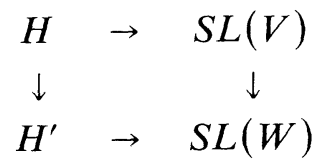

commutes.

Let $T$ be the one dimensional subtorus of $H$ of diagonal matrices of the form: $\operatorname{diag}\left(a, \ldots, a, a^{1-n}\right)$ for $a \in K^{\times}$. Let $S$ be the group of scalar matrices from $G L(V)$, so $\tau(T)=T S / S$. Choosing a complement for $S$ in the two dimensional torus TS shows that the comorphism of $\tau$ identifies the coordinate ring of $\tau(T)$ with $K\left[x^{n}, x^{-n}\right]$, where $K[T]=K\left[x, x^{-1}\right]$. So our preceding discussion shows how $\tau(T)$ may be expressed as a quotient of $T$. If $X$ is a generator for $L(T)$, then $X^{(l)}\left(i<p^{s}\right)$ lies in the kernel of $h y(\tau)$ while the image of $X^{(i)}\left(q=p^{s}\right)$ generates $L(\tau(T))$. The commutativity of (4.1) shows that $L(\tau(T))$ acts on $W$ by a non-trivial linear character. Since $\operatorname{Im} d \tau$ annihilates $W$ we have, for dimensional reasons, $\mathfrak{h}^{\prime}=\operatorname{Im} d \tau \oplus L(\tau(T))$.

Now define a sequence of purely inseparable quotient morphisms $H_{l} \rightarrow H_{i+1}$ as follows. Let $H_{0}=H$ and $T_{0}=T$. For $i<s$, set $H_{t+1}=$ $H_{l} / L\left(T_{l}\right)$ and let $T_{l+1}=\mu_{i}\left(T_{i}\right)$, where $\mu_{t}: H_{l} \rightarrow H_{l+1}$ is the quotient morphism. To show the existence of these quotient groups it suffices to show that $L\left(T_{i}\right)$ is central in $\mathfrak{h}_{i}$.

Since we are assuming $s>0, L\left(T_{0}\right)$ is central in $\mathfrak{h}_{0}$. Assume the result for $i-1$ and form the quotient group $H_{i}$. Then $V^{(l)}$ is a rational 
$H_{i}$-module and $\tau$ factors through $H_{i}$ to yield a group morphism $\tau_{i}$ : $H_{i} \rightarrow H^{\prime}$ for which the diagram

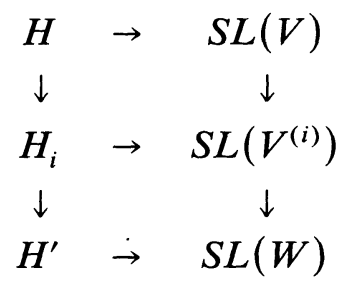

commutes. Then $L\left(T_{i}\right)$ is generated by the image of $X^{(q)}\left(q=p^{i}\right)$ which acts non-trivially on $V^{(i)}$. Since $\operatorname{Im} d \mu_{i-1}$ annihilates $V^{(i)}$ we have, again for dimensional reasons, $\mathfrak{h}_{i}=\operatorname{Im} d \mu_{i-1} \oplus L\left(T_{i}\right)$.

If $i<s$, then $X^{(q)}$ lies in the kernel of $h y(\tau)$ and so $L\left(T_{i}\right)$ is contained in the kernel of $d \tau_{i}$. A dimension comparison shows that the two are equal and hence $L\left(T_{i}\right)$ is central in $\mathfrak{h}_{i}$. On the other hand, $L\left(T_{s}\right)$ is not contained in the kernel of $d \tau_{s}$. Consequently,

$$
\mathfrak{h}^{\prime}=\operatorname{Im} d \tau \oplus L(\tau(T))=\operatorname{Im} d \tau_{s}
$$

and so $\tau_{s}: H_{s} \rightarrow H^{\prime}$ is a surjective separable morphism. If $M$ is the kernel of $\tau_{s}$, then $M$ is isomorphic to the group of $m$ th roots of unity in $K$ and $H_{s} / M \cong H^{\prime}$.

In case $s=0$, we have $H_{s}=H$ and $H / M \cong H^{\prime}$.

REMARK. Each $H_{i}$ is, in the context of Chevalley groups, the group corresponding to the lattice of weights determined by $p^{i} \lambda$, where $\lambda$ is a fixed dominant weight. The inseparable morphisms $H_{i} \rightarrow H_{i+1}$ alter the structure constants of the respective Lie algebras to yield a group $H_{s}$ whose Lie algebra is center free. The group $H^{\prime}$ is the Chevalley group corresponding to the lattice of weights determined by $m p^{s} \lambda=n \lambda$. From the viewpoint of group schemes, $H^{\prime}$ is the quotient of $H$ by the diagonalizable group scheme represented by $u_{s}(T)^{*} \times K[M]^{*}$, where $K[M]$ is the group algebra of $M$.

(4.3) Lemma. Assume that $\operatorname{Hom}\left(\mathfrak{g}, \mathfrak{g}_{m}\right)=0$ and let $\pi: G \rightarrow G L(V)$ be a rational projective representation. Then there is a connected affine algebraic group $G(V)$ and a rational representation $\rho: G(V) \rightarrow S L(V)$ such that

(a) $G(V)$ has a finite central $p^{\prime}$-subgroup $N$ with $G(V) / N \cong G$, and

(b) the following diagram is commutative,

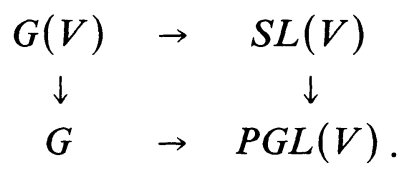


Proof. Let $H=S L(V), H^{\prime}=P G L(V)$ and write $\operatorname{dim} V=p^{s} m$. Retaining the notation of (4.2) we define $G_{s}$ as the connected component of the identity in the fibred product of $\pi^{\prime}: G \rightarrow H^{\prime}$ and $\tau_{s}: H_{s} \rightarrow H^{\prime}$. Let $N$ be the kernel for the projection of $G_{s}$ onto $G$. Then $N=(1 \times M) \cap G_{s}$ is a central $p^{\prime}$-subgroup of $G_{s}$ with $G_{s} / N \cong G$.

Composing $\pi^{\prime}$ with a power of the Frobenius map $F$ yields a morphism $F^{\prime} \pi^{\prime}: G \rightarrow P G L\left(V^{(l)}\right)$. We show, by descending induction on $i$, the existence of group morphisms $\rho_{l}: G_{s} \rightarrow S L\left(V^{(t)}\right)$ such that the following diagram is commutative

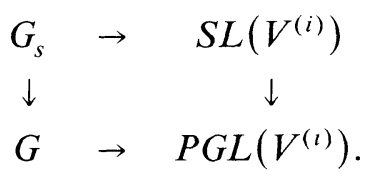

The lemma follows by setting $G(V)=G_{s}$ and $\rho=\rho_{0}$.

For $i=s$, we define $\rho_{s}$ as the projection of $G_{s}$ into $H_{s}$ composed with the representation of $H_{s}$ in $S L\left(V^{(s)}\right)$ given in (4.2). Assume the existence of $\rho_{i}(0<i \leq s)$ and let $\beta_{l}: S L\left(V^{(i)}\right) \rightarrow P G L\left(V^{(i)}\right)$ be the quotient morphism. If $\alpha: G_{s} \rightarrow G$ denotes the projection map, then our induction hypothesis shows that $\beta_{i} \rho_{t}=F^{\prime} \pi^{\prime} \alpha$ and consequently $d \beta_{i} d \rho_{i}=0$. Hence $\operatorname{Im} d \rho_{l} \leq \operatorname{Ker} d \beta_{i}$ which is either 0 or $\mathfrak{g}_{m}$. Since $\operatorname{Hom}\left(g, \mathfrak{g}_{m}\right)=0, \operatorname{Im} d \rho_{l}$ $=0$. So there is a group morphism $\rho_{l-1}: G_{s} \rightarrow S L\left(V^{(2-1)}\right)$ such that $F \circ \rho_{l-1}=\rho_{l}$. The commutativity of the corresponding diagram follows from the choice of $\rho_{i-1}$.

Assume that $\operatorname{Hom}\left(g, g_{m}\right)=0$ and let $W_{l}(i \in I)$ be a set of representatives for the isomorphism classes of irreducible restricted representations of $\mathrm{g}$. Each $W_{i}$ induces a rational projective representation of $G$ and hence, by Lemma 4.3 , yields a separable isogeny $G\left(W_{t}\right) \rightarrow G$. This isogeny is an isomorphism if and only if $W_{i}$ is a linear $G$-module. The differential of the representation $G\left(W_{l}\right) \rightarrow S L\left(W_{i}\right)$ agrees with the original representation of $\mathrm{g}$ on $W_{i}$. We define $G^{s}$, the Schur representation group of $G$ relative to $\mathfrak{g}$, as the connected component of the identity in the fibred product of the $G\left(W_{l}\right) \rightarrow G(i \in I)$. The definition of $G^{s}$ shows that every irreducible representation of $\mathfrak{g}$ is the differential of a uniquely determined rational representation of $G^{s}$. In fact, Theorem 3.1 and Lemma 4.3 insure that every rational projective representation of $G^{s}$ is equivalent to a rational linear representation. Define the relative Schur multiplier $M(G)$ as the character group for the kernel of the map $G^{s} \rightarrow G$. 
(4.4) Theorem. Assume that $\operatorname{Hom}\left(g, g_{m}\right)=0$. The irreducible rational projective representations of $G$ are, modulo projective equivalence, given by

$$
W_{0} \otimes W_{1}^{(1)} \otimes \cdots \otimes W_{n}^{(n)}
$$

where the $W_{i}$ are irreducible restricted representations of $\mathrm{g}$.

Proof. Since equivalent linear representations of $G^{s}$ yield equivalent projective representations of $G$ and $L\left(G^{s}\right)=L(G)$, we may assume that $G^{s}=G$.

Let $V$ be an irreducible rational $G$-module. We proceed by induction on the dimension of $V$. If $V \mid \mathrm{g}$ is trivial, then there is an integer $n$ and an irreducible $G$-module $V_{1}$ such that $V=V_{1}^{(n)}$ and $V_{1} \mid \mathfrak{g}$ is non-trivial. Replacing $V$ by $V_{1}$ shows that we may assume $V$ has a non-trivial g-submodule $W_{0}$. Now $W_{0}$ is a linear $G$-module and so by Theorem 3.1 there is an irreducible rational $G$-module $U_{1}$ with $V \cong W_{0} \otimes U_{1}^{(1)}$. Then $\operatorname{dim} U_{1}<\operatorname{dim} V$ and so our induction hypothesis shows that

$$
U_{1} \cong W_{1} \otimes W_{2}^{(1)} \otimes \cdots \otimes W_{n}^{(n-1)}
$$

for certain irreducible representations $W_{i}$ of $\mathrm{g}$. Since $V \cong W_{0} \otimes U_{1}^{(1)}$, we have the desired factorization.

We now show that any projective representation of the form given by 4.5 is irreducible. Since we are assuming that $G=G^{s}$, the representations in question are actually linear. We proceed by induction on $n$. Let

$$
V=\bigotimes_{i=0}^{n} W_{i}^{(i)} \text { and } U=\bigotimes_{i=1}^{n} W_{i}^{(i-1)}
$$

Then $V \cong W_{0} \otimes U^{(1)}$ and our induction hypothesis shows $U$ is irreducible. Suppose that $V_{1}$ is an irreducible $G$-submodule of $V$. Then $W_{0}$ is a g-submodule of $V_{1}$ and hence by Theorem 3.1; $V_{1} \cong W_{0} \otimes U_{1}^{(1)}$. But

$$
U_{1}^{(1)} \cong \operatorname{Hom}_{\mathfrak{g}}\left(W_{0}, V_{1}\right) \text { and } U^{(1)} \cong \operatorname{Hom}_{\mathfrak{g}}\left(W_{0}, V\right)
$$

with the former a $G$-submodule of the latter. So $U_{1}$ is a $G$-submodule of $U$, contradicting the irreducibility of $U$.

We now show that the irreducible linear representations of the infinitesimal hyperalgebras are determined by the representations of the Lie algebra. In case $G$ is simply connected and semisimple, this result is due to Humphreys [10].

(4.6) Corollary. If $\operatorname{Hom}\left(\mathfrak{g}, g_{m}\right)=0$, then the irreducible linear representations of $u_{m}$ are given by equation (4.5) with $n=m-1$. 
Proof. The differential of the quotient morphism $G^{s} \rightarrow G$ is an isomorphism of Lie algebras and hence yields an isomorphism of the respective infinitesimal hyperalgebras. So we may assume that $G=G^{s}$. We prove the result for $m=2$ and note that the general case is similar.

Let $W$ be an irreducible linear representation of $u_{2}$. Then $W$ induces a projective representation of $G$ which, since $G=G^{s}$, is actually a linear representation. By Theorem 4.4,

$$
W \cong W_{0} \otimes W_{1}^{(1)} \otimes \cdots \otimes W_{n}^{(n)}
$$

and since $W \mid u_{2}$ is irreducible, the $W_{l}$ for $i>2$ are trivial.

Now suppose that $W_{0}$ and $W_{1}$ are irreducible representations of $g$ and hence of $G$. Let $W$ be an irreducible $u_{2}$-submodule of $W_{0} \otimes W_{1}^{(1)}$. By the first part of the proof, $W \cong W_{0} \otimes W_{2}^{(1)}$ for some irreducible representation $W_{2}$ of $g$. Arguing as in the proof of Theorem 4.4 shows that $W_{2}$ is isomorphic to a $g$-submodule of $W_{1}$ and hence completes the proof of the corollary.

EXAMPle. Let $p=2$ and let $W$ be a two dimensional vector space over $K$. If $G=P G L(W)$, then $V=W^{(1)}$ is an irreducible linear $G$-module. For $\chi$ the non-trivial linear character of $g, S(\chi)$ is a $g$-submodule of $V$. Since $g$ has no irreducible two dimensional representations, it is not possible to factor $V$ as in Theorem 4.4. We note that $V$ is an irreducible $u_{2}$-module and so (4.4) and (4.6) may both fail if $\operatorname{Hom}\left(\mathfrak{g}, \mathfrak{g}_{m}\right) \neq 0$.

5. Identifications. We begin the identification of $G^{s}$ and $M(G)$ by summarizing the connection between the projective representations of $G$ and its Picard group, referring the reader to [8] for details. Let $B$ be a Borel subgroup of $G$ and let $D$ be a positive divisor on $G / B$. Since $G / B$ is a projective variety, the linear system corresponding to $D$ is finite dimensional and hence of the form $\mathbf{P}(V)$, for some vector space $V$. By [11], $G$ acts rationally on $\mathbf{P}(V)$ and consequently yields a rational projective representation of $G$. This representation is equivalent to a linear one if and only if the image of $D$ in the group of divisors on $G$ is linearly equivalent to 0 . Since the morphism $G \rightarrow G / B$ induces a surjection of Picard groups $\operatorname{Pic}(G / B) \rightarrow \operatorname{Pic}(G)$, we see that $M(G)=0$ implies $\operatorname{Pic}(G)$ $=0$. The converse is contained in the following corollary.

(5.1) Corollary. Assume that $\operatorname{Hom}\left(g, g_{m}\right)=0$. Then the relative Schur covering group $G^{s}$ is the simply connected covering group of $G$ and $\operatorname{Pic}(G) \cong M(G)$. 
Proof. Our construction of $G^{s}$ shows that every rational projective representation is linearizable. So $G^{s}$ is simply connected in the sense of representation groups. To see that $G^{s}$ is simply connected as an algebraic variety, we note that $M\left(G^{s}\right)=0$ and hence $\operatorname{Pic}\left(G^{s}\right)=0$.

Let $N$ be the kernel of the covering morphism $G^{s} \rightarrow G$. Set $A=K\left[G^{s}\right]$ and let $\mathrm{Cl}$ denote the divisor class group. Then $K[G]=A^{N}$ and so $\operatorname{Pic}(G)=\operatorname{Cl}\left(A^{N}\right)$ which we identify by Galois descent $[9$, p. 82] from $A$ to $A^{N}$.

Let $P$ be a prime ideal of $A^{N}$. Since $\mathrm{Cl}(A)=\operatorname{Pic}\left(G^{s}\right)=0, P A=A a$ for some $a \in A$. Let $Q$ be the quotient field of $A$ and let $P(A)$ denote the group of principal divisors $A x$ for $x \in Q$. If Div denotes the group of divisors, then the map $P \rightarrow P A$ yields an injection $\operatorname{Div}\left(A^{N}\right) \rightarrow P(A)^{N}$. Realizing $A$ as the quotient of a polynomial ring shows that $A$ is unramified over $A^{N}$ and it follows that this map is surjective. Passing to the class group yields an isomorphism $\mathrm{Cl}\left(A^{N}\right) \cong P(A)^{N} / P\left(A^{N}\right)$, where $P\left(A^{N}\right)$ denotes the subgroup of principal divisors $A x$ for $x \in Q^{N}$.

Write $Q=\oplus Q(\mu)$, where $Q(\mu)$ is the space of semi-invariants of weight $\mu$, for $\mu \in \operatorname{Hom}\left(N, K^{\times}\right)$. Our assumption that $\mathfrak{g}$ is character free insures that $G^{s}$ is also and hence $K^{\times}$is the group of units of $A$ [12]. Consequently, $P(A)^{N}$ consists of the principal divisors $A x$ for $x \in Q(\mu)$ with $A x \equiv A y$, modulo $P\left(A^{N}\right)$, if and only if $x y^{-1} \in Q(1)=Q^{N}$.

Now let $R\left(G^{s}\right)$ be the Grothendieck group, or formal character group, for the irreducible rational representations of $G^{s}$ and view $R(G)$ as a subgroup. Let $V$ be an irreducible rational representation space for $G^{s}$ and let $a \in A$ denote any coordinate function for the representation. Since $N$ is central in $G^{s}, a \in A(\mu)$ for some $\mu$ which is independent of the choice of the coordinate function. Let $[V] \in P(A)^{N} / P\left(A^{N}\right) \cong \operatorname{Pic}(G)$ be the principal divisor $A a$. Then $[V]=A$ if and only if $V$ is a linear representation of $G$ and so we obtain an injection

$$
R\left(G^{s}\right) / R(G) \rightarrow \operatorname{Pic}(G)
$$

which it is easy to see is surjective.

By Theorem 4.4

$$
V=\bigotimes_{i=0}^{n} W_{i}^{(i)}, \text { and hence }[V]=\prod_{i=0}^{n}\left[W_{l}\right]^{p^{i}} .
$$

So the $[W]$, for $W$ an irreducible representation of $g$ which is not the differential of a rational representation for $G$, generate $\operatorname{Pic}(G)$. Noting that $V \mid N$ affords some $\mu \in \operatorname{Hom}\left(N, K^{\times}\right)=M(G)$ completes the identification. 


\section{REFERENCES}

1. A. Bialynicki-Birula, G. Hochschild, and G. Mostow, Extensions of representations of algebraic linear groups, Amer. J. Math., 85 (1963), 131-144.

2. A. Borel, Linear Algebraic Groups, Benjamin, New York, 1969.

3. S_ Seminar on Algebraic Groups and Related Finite Groups, Part A, Lecture Notes in Mathematics No. 131, Springer, Berlin, 1970.

4. A. H. Clifford, Representations induced in an invariant subgroup, Ann. of Math., 38 (1937), 533-550.

5. E. Cline, B. Parshall, and L. Scott, On the tensor product theorem for algebraic groups, J. Algebra, 63 (1980), 264-267.

6. C. W. Curtis, Representations of Lie algebras of classical type with applications to linear groups, J. Math. Mech., 9 (1960), 307-326.

7. C. W. Curtis and I. Reiner, Representation Theory of Finite Groups and Associative Algebras, Wiley, New York, 1962.

8. R. Fossum and B. Iversen, On Picard groups of algebraic fibre spaces, J. Pure Appl. Algebra, 3 (1973), 269-280.

9. R. Fossum, The Divisor Class Group of a Krull Domain, Springer, Berlin, 1973.

10. J. E. Humphreys, On the hyperalgebra of a semisimple algebraic group, Contributions to Algebra: A Collection of Papers Dedicated to Ellis Kolchin, Academic Press, New York, 1977.

11. T. Kambayashi, Projective representations of algebraic linear groups of transformations, Amer. Math. J., 88 (1966), 199-205.

12. M. Rosenlicht, Toroidal algebraic groups, Proc. Amer. Math. Soc., 12 (1961), 984-988.

13. J. Sullivan, Representations of the hyperalgebra of a semisimple group, Amer. J. Math., 100 (1978), 643-652.

Received September 28, 1981. Research supported by the National Science Foundation.

UNIVERSITY OF WASHINGTON

SEATtLE, WA 98195 



\section{PACIFIC JOURNAL OF MATHEMATICS EDITORS}

DONALD BABBITT (Managing Editor)

University of California

Los Angeles, CA 90024

Hugo Rossi

University of Utah

Salt Lake City, UT 84112

C. C. Moore and Arthur Ogus

University of California

Berkeley, CA 94720
J. DugunduI

Department of Mathematics

University of Southern California

Los Angeles, CA 90089-1113

R. FinN and H. SAmelson

Stanford University

Stanford, CA 94305

\section{ASSOCIATE EDITORS}
R. ARENS
E. F. BECKENBACH
B. H. NeUmaNN
F. WolF
K. YoshidA (1906-1982)

\section{SUPPORTING INSTITUTIONS}

UNIVERSITY OF ARIZONA

UNIVERSITY OF BRITISH COLUMBIA

CALIFORNIA INSTITUTE OF TECHNOLOGY

UNIVERSITY OF CALIFORNIA

MONTANA STATE UNIVERSITY

UNIVERSITY OF NEVADA, RENO

NEW MEXICO STATE UNIVERSITY

OREGON STATE UNIVERSITY
UNIVERSITY OF OREGON

UNIVERSITY OF SOUTHERN CALIFORNIA

STANFORD UNIVERSITY

UNIVERSITY OF HAWAII

UNIVERSITY OF TOKYO

UNIVERSITY OF UTAH

WASHINGTON STATE UNIVERSITY

UNIVERSITY OF WASHINGTON 


\section{Pacific Journal of Mathematics}

\section{Vol. 106, No. $1 \quad$ November, 1983}

John Ballard, Clifford's theorem for algebraic groups and Lie algebras . . . . . 1

Philip Richard Bartick, II and Edwin Duda, Finite-to-one open mappings

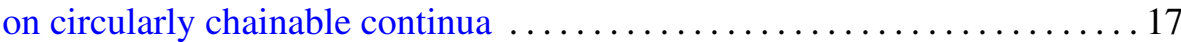

Frank Hayne Beatrous, Jr., $H^{\infty}$-interpolation from a subset of the

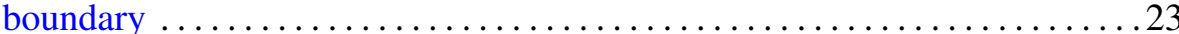

Sterling K. Berberian, Expectations in semifinite algebras ............ 33

Heron S. Collins and Wolfgang Ruess, Weak compactness in spaces of compact operators and of vector-valued functions $\ldots \ldots \ldots \ldots \ldots \ldots . \ldots 45$

David Downing and William O. Ray, Renorming and the theory of

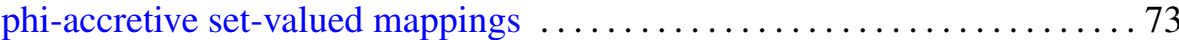

Harvey Charles Greenwald, On the theory of homogeneous Lipschitz

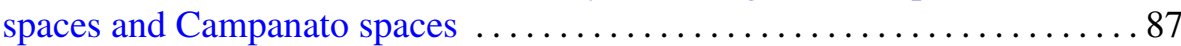

Irwen Valle Guadalupe and Lucio Ladislao Rodriguez, Normal curvature

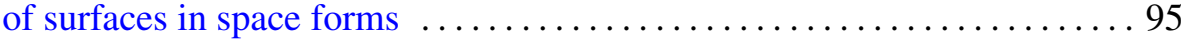

Boju Jiang, On the computations of the Nielsen number ............... 105

William H. Julian, Ray Mines, III and Fred Richman, Alexander

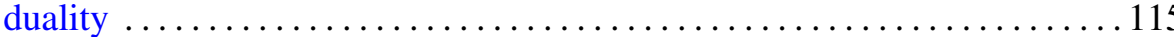

Midori Kobayashi, The connected component of the idèle class group of an

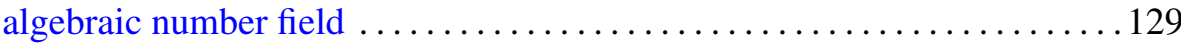

Ib Henning Madsen, Charles B. Thomas and C. Terence C. (Charles)

(Clegg) Wall, Topological spherical space form problem. III.

Dimensional bounds and smoothing

A. Sapounakis, The existence of strong liftings for totally ordered measure

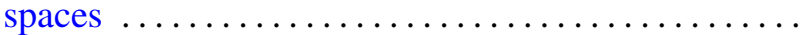

Gary Seitz, The root subgroups for maximal tori in finite groups of Lie type

Laurent Siebenmann and James M. Van Buskirk, Construction of irreducible homology 3-spheres with orientation reversing involution 